\title{
Review
}

\section{Obesity: modern man's fertility nemesis}

\author{
Stephanie Cabler ${ }^{1}$, Ashok Agarwal ${ }^{1}$, Margot Flint ${ }^{2}$, Stefan S. Du Plessis ${ }^{2}$ \\ ${ }^{1}$ Center for Reproductive Medicine, The Cleveland Clinic, 9500 Euclid Avenue, Cleveland, OH 44195, USA \\ ${ }^{2}$ Division of Medical Physiology, Faculty of Health Sciences, Stellenbosch University, PO Box 19063, Tygerberg 7505, South Africa
}

\begin{abstract}
The obesity pandemic has grown to concerning proportions in recent years, not only in the Western World, but in developing countries as well. The corresponding decrease in male fertility and fecundity may be explained in parallel to obesity, and obesity should be considered as an etiology of male fertility. Studies show that obesity contributes to infertility by reducing semen quality, changing sperm proteomes, contributing to erectile dysfunction, and inducing other physical problems related to obesity. Mechanisms for explaining the effect of obesity on male infertility include abnormal reproductive hormone levels, an increased release of adipose-derived hormones and adipokines associated with obesity, and other physical problems including sleep apnea and increased scrotal temperatures. Recently, genetic factors and markers for an obesity-related infertility have been discovered and may explain the difference between fertile obese and infertile obese men. Treatments are available for not only infertility related to obesity, but also as a treatment for the other comorbidities arising from obesity. Natural weight loss, as well as bariatric surgery are options for obese patients and have shown promising results in restoring fertility and normal hormonal profiles. Therapeutic interventions including aromatase inhibitors, exogenous testosterone replacement therapy and maintenance and regulation of adipose-derived hormones, particularly leptin, may also be able to restore fertility in obese males. Because of the relative unawareness and lack of research in this area, controlled studies should be undertaken and more focus should be given to obesity as an etiolgy of male infertility.
\end{abstract}

Asian Journal of Andrology (2010) 12: 480-489. doi: 10.1038/aja.2010.38; published online 7 June 2010.

Keywords: adipokines, infertility, leptin, obesity, spermatozoa

\section{Introduction}

Obesity has become an epidemic not only in the United States, but increasingly so in the rest of the world. The world's overweight population is already greater than its underweight population and the numbers of overweight individuals are rapidly on the increase,

Correspondence to: Prof. Ashok Agarwal, Center for Reproductive Medicine, The Cleveland Clinic, 9500 Euclid Avenue, Cleveland, $\mathrm{OH} 44195$, USA.

Fax: +1-21-6445-6049

Received: 1 February 2010

Accepted: 14 April 2010
E-mail: agarwaa@ccf.org

Revised: 31 March 2010

Published online: 7 June 2010 leading to a global obesity pandemic [1]. Once considered a problem only in high-income countries, overweight and obesity are now dramatically on the rise in all countries. This is evident in the $5 \mathrm{U}$ increase in body mass index (BMI) for the period 1997-2006 in the 95th centile BMI level among children aged 6-9 years in China. The children in the 95th centile have a BMI of 24.8, which is surprisingly higher than that of those in the United States (22.2), Australia (20.1) and the United Kingdom (20.1) [2]. According to the World Health Organization, in 2006, the prevalence of obesity or morbid obesity in the United States was $41.3 \%$ [3]. It is predicted that globally, in the next 5 years, more than 700 million adults will suffer from obesity [3]. 
These shifts, specifically among children, suggest that debility, disability and societal costs due to obesity are also dramatically on increase [2].

Parallel to the global increase in obesity is the reported decrease in male fertility and fecundity [4]. Interestingly enough, men with increased BMI were significantly more likely to be infertile than normal weight men, according to a research conducted at the National Institute of Environmental Health Sciences [5].

More scholarly reports are documenting that the decline in semen quality and male reproductive potential over the past half century may be a result of the increasing incidence of obesity. According to Carlsen et al. [6], there has been a substantial decline in semen quality, and because of the effect poor semen quality has on male fertility, plausibly an overall decrease in male reproductive potential. Some studies estimate that male sperm counts continue to decrease by as much as $1.5 \%$ per year in the United States. These findings are similar to those found in other Western countries and countries in which obesity is more prevalent, but are not present in regions in which obesity is less common [4]. In addition, there is also a significant increase in the incidence of obesity in patients with male-factor infertility, and couples with obese male partners are more likely to experience subfecundity, a correlation that seems necessary to address [7]. Owing to the fact that this decline has occurred relatively in parallel with increasing rates of obesity, it is necessary to focus on the possibility of obesity as an etiology of male infertility and reduced fecundity. The 'obesity pandemic' seen in many countries is a serious threat to public health, and a reduced capacity to reproduce is a potential but less wellknown health hazard attributed to obesity.

Therefore, the purpose of this review is to explore the links between obesity and male infertility, to address how it disrupts the male reproductive system at a mechanistical level, and to discuss the treatment and prevention of obesity and associated fertility disorders.

\section{What is obesity?}

The fundamental cause of obesity and overweight is an energy imbalance, wherein the energy consumed exceeds the energy expended. Contemporary lifestyles and the accompanying pressures have been implicated extensively as the root cause of this global phenomena experienced by modern man. Global increases in overweight and obesity are attributable to a number of factors including a shift in diet toward increased intake of energy-dense foods that are high in fat and sugars and a trend toward decreased physical activity, as a result of increasingly sedentary nature of work, changing modes of transportation and increasing urbanization.

Traditionally, obesity has been defined as a weight at least $20 \%$ above the weight corresponding to the lowest death rate for individuals of a specific height, gender and age [3]. Currently, overweight and obesity are defined more broadly as abnormal or excessive fat accumulation that may impair health. However, there are other specific requirements that qualify an individual as obese. Measuring the exact amount of a person's body fat is not easy. The most accurate measures are to weigh a person underwater or to use an X-ray test called Dual energy X-ray absorptiometry. These methods are not practical for an average individual, and are conducted only in research centers with special equipment. There are simpler methods to estimate body fat such as BMI, skinfold measurements, waist to hip ratio (WHR), waist circumference, and also methods such as bioelectrical impedance analysis, risk factors and comorbidities [8]. BMI and waist circumference are two most commonly used to identify obese patients. BMI is a simple index of the weight-to-height ratio that is commonly used in classifying overweight and obesity in adult populations and individuals. It is defined as the weight in kilograms divided by the square of the height in meters $\left(\mathrm{kg} \mathrm{m}^{-2}\right)$. A BMI of greater than $25 \mathrm{~kg} \mathrm{~m}^{-2}$ is considered overweight and greater than $30 \mathrm{~kg} \mathrm{~m}^{-2}$ is considered as obese. A problem with this method is that individuals with a high BMI may be mesomorphic and have a high amount of muscle mass. Therefore, BMI may not be the most accurate marker for total body fat percentage and an even less suitable tool to assess body fat distribution. Waist circumference is a slightly less common method used to predict obesity in an individual, but may be more accurate in predicting obesity-related health issues. For females, a waist circumference of $88 \mathrm{~cm}$ or greater is considered unhealthy. For men, a waist circumference of $102 \mathrm{~cm}$ or greater is considered unhealthy. If waist circumference is used as the criterion, then according to a study conducted in 2006, the prevalence of being overweight among Australian adults, and probably among other Caucasian populations, may be significantly greater than indicated by surveys relying on self-reported height and weight [9]. The development of valid self-reported measures of waist circumference for use in population surveys 
may allow more accurate monitoring of overweight and obesity, and perhaps should be considered instead of BMI [9]. A WHR can also be used to predict unhealthy consequences as a result of increased body fat (normal WHR: males $\leq 0.9$; females $<0.85$ ), especially those related to the risk of coronary heart disease, as it relates to obesity [10]. Waist circumference, BMI and WHR can all identify different proportions of the population, as measured for obesity prevalence. The most common methods for diagnosing overweight and obesity are based on BMI $\left(\mathrm{kg} \mathrm{m}^{-2}\right)$. WHR may be the most useful measure of obesity and the best simple anthropometric index in predicting a wide range of risk factors and related health conditions [11]

\section{How does obesity affect male fertility?}

Obesity is a serious, chronic disease with several comorbidities that include cancer, non-insulindependant diabetes mellitus, gallbladder disease, high cholesterol, atherosclerosis, heart disease, hypertension, stroke, psychological depression, sleep apnea and renal failure $[12,13]$.

Significant quantities of data show a relationship between obesity and subfecundity, thereby establishing obesity as a further comorbidity [5]. Basically, obesity impinges on the male reproductive system and fertility through its effects on erectile dysfunction (ED) and impaired semen parameters (Figure 1).

ED is a common reported cause of infertility or reproductive difficulties in obese and diabetic males. Several scholars have reported a correlation between obesity and ED [14]. Corona et al. [15] presented evidence showing that $96.5 \%$ of their subjects with metabolic syndrome, which is characteristic of abdominal obesity, exhibited ED. A directly proportional relationship between the increasing severity of obesity and the severity of ED was reported.

Overweight and obese couples are at a higher risk of being subfecund. If both partners are obese, they face a higher risk of fertility problems than if only one partner was diagnosed as being obese [16]. This points to both a female and male factor that could contribute to infertility, and also draws attention to a plausible explanation for male-factor infertility.

Altered semen parameters ascribed to obesity include decreased sperm concentration, abnormal morphology, compromised chromatin integrity and abnormal motility. Although there is a convincing

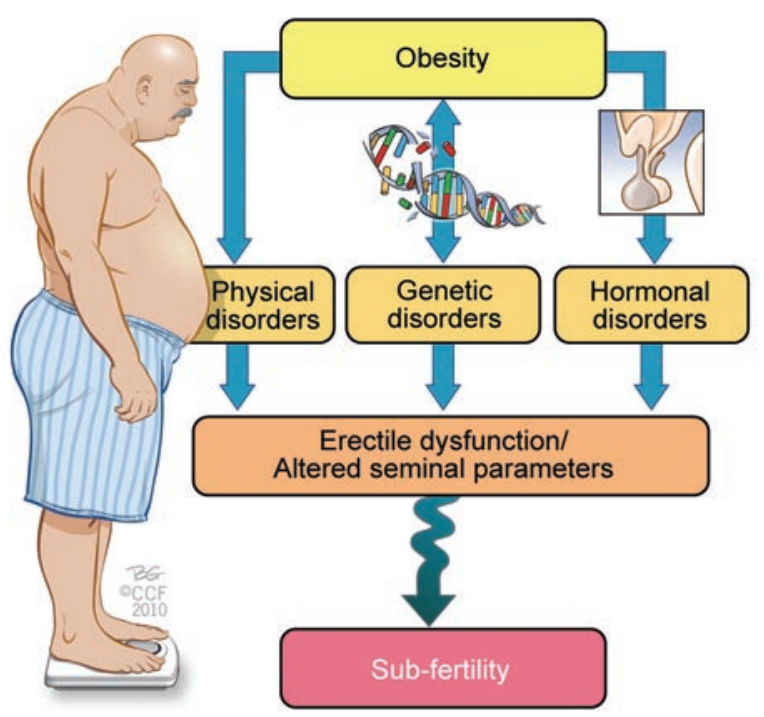

Figure 1. The effects of obesity on male infertility and the mechanisms involved.

amount of evidence to show an adverse affect of excess body fat on spermatogenesis, not all studies have come to the same conclusions $[4,9,17-20]$.

In one of the largest studies carried out on Danish men, Jensen et al. [17] measured BMI in relation to semen quality and reproductive hormones and found some significant relationships between sperm concentration and BMI. A lower sperm concentration was observed in not only obese and overweight males but also in males who were significantly underweight. This could serve as an indication that there may be an ideal range of BMI for normal spermatogenesis. Subjects whose BMI was within the normal range showed a higher sperm concentration, as well as a higher total sperm count and a lower percentage of abnormal spermatozoa. In this particular study, BMI was not associated with poor sperm morphology or motility, but they did suggest that obesity could be an explanation for the subfertility of the Danish population and of the Western world in general. Another study by Martini et al. [18] found that there was a negative association between BMI and motility, rapid motility, and a positive association between BMI and seminal fructose levels. No associations were found among BMI and sperm concentration or seminal testosterone levels.

A 2008 study performed by Hammoud et al. [19] also found that among their 526 patients, the prevalence of a low progressively motile sperm count and furthermore low 
sperm concentration was greater with increasing BMI.

DNA fragmentation is a common abnormal sperm parameter observed in obese males, and, although it can lead to fertility problems alone, it may also indicate a poor quality of overall spermatogenesis, which could also affect the concentration and motility of sperm [20]. This leads one to believe that not only do obese males have altered semen parameters but the root of these problems could also be a result of abnormal spermatogenesis, perhaps due to an altered hormonal profile .

A new breakthrough in gel electrophoresis has been used to identify obesity-associated changes of the sperm proteome. Semen samples from obese males differed from those of normal weight men, with 12 spots seen after running their 'difference gel electrophoresis' of fluorescently labeled human sperm proteins. Tryptic digestion of the 12 spot proteins and mass spectrometric analysis of the corresponding peptides identified nine sperm proteins apparently associated with obesity. This can now be considered as a noninvasive experimental tool in the diagnosis of male infertility and in monitoring any fertility-restoring therapy in obese males [21]. This concrete information clearly displays the differences or changes in the protein composition of sperm in obese men.

\section{What are the pathophysiological mechanisms?}

Several underlying pathophysiological mechanisms can link obesity to male-factor infertility. It would be impossible to discuss all of them in detail and therefore, for the purpose of this review, these factors are grouped and discussed briefly.

\subsection{Genetic factors}

Klinefelter, Prader-Willi and Laurence-MoonBardet-Biedel syndromes, due to genetic mutations, all exhibit to certain extent levels of both obesity and infertility [22]. Prader-Willi syndrome is an extreme genetic example of a syndrome that has symptoms of both obesity and infertility, due to an abnormality on chromosome 15. In addition, mutations in the human ALMS1 gene are responsible for Alström syndrome, a disorder in which key metabolic and endocrinological features include childhood-onset obesity, metabolic syndrome and diabetes, as well as infertility [23]. Scientists still need to accurately establish which particular genes are involved in these different syndromes, but the linkage of obesity and infertility in the extreme points to some genetic linkage. Hammoud et al. [24] recently discovered that an aromatase polymorphism modulates the relationship between weight and estradiol levels in obese men. This could explain why only certain obese men experience this rise in estradiol and subsequent fertility problems, whereas others experience no fertility issues. It seems possible that there are other less severe genetic markers than chromosome 15 abnormalities and ALMS1 mutations that may clarify the discrepancies between obese fertile and infertile men, and give explanation to a possible genetic link between obesity and infertility.

\subsection{Hormonal mechanisms and adipokines}

Abdominal or visceral fat is more likely to lead to changes in hormone levels and to cause inflammation than fat stored in other parts of the body. This is predominantly due to the fact that white adipose tissue, found in high levels in obese men, exhibits elevated aromatase activity and secretes adipose-derived hormones as well as adipokines.

The reproductive hormonal profiles of most obese men deviate from what is considered the norm. Obese men tend to present with elevated estrogen and low testosterone and FSH levels. Androgen deficiency or hypogonadism found in males who are obese or have metabolic syndrome can account for problems with ED and spermatogenesis. However, many other hormones associated with obesity may alter the male reproductive potential. In morbidly obese individuals, reduced spermatogenesis associated with severe hypotestosteronemia may contribute to infertility [25, 26]. This estrogen excess is explained by overactivity of the aromatase cytochrome P450 enzyme, which is expressed at high levels in white adipose tissue and is responsible for a key step in the biosynthesis of estrogens. High levels of estrogens in obese males result from the increased conversion of androgens into estrogens, owing to the high bioavailability of these aromatase enzymes [27]. Visceral obesity can serve as a major endocrine disrupter and can also influence the endocrine interactions by reducing the levels of luteinizing hormone (LH) and testosterone, resulting in hypogonadotropic hypogonadism, a condition that contributes to male infertility. Although abnormal levels of reproductive hormones could be the source of fertility problems in obese males, Qin et al. [28] established that the associations between BMI and 
semen quality were found to be statistically significant even after an adjustment for reproductive hormones, thereby showing that reproductive hormones cannot fully explain the association between BMI and semen quality. Perhaps, altered hormone levels themselves do not explain poor semen quality, but instead a deregulation of the normal hypothalamic-pituitary-gonadal (HPG) axis. Regardless, studies indicate that the association between BMI and semen quality is clearly more complex than what can be accounted for simply by reproductive hormones. Instead it may be a result of other factors such as one's lifestyle and increased adipokine release.

White adipose tissue is a major secretory and endocrine organ that secretes $\sim 30$ biologically active peptides and proteins that can be grouped as either adipose-derived hormones (for example, leptin, adiponectin and resistin) or adipokines (immunomodulating agents). Adipose-derived hormones have a central role in body homeostasis, including the regulation of food intake and energy balance, insulin action, lipid and glucose metabolism, angiogenesis and vascular remodeling, coagulation and in the regulation of blood pressure [29]. Owing to an excess of white adipose tissue in obese men, levels of adipose-derived hormones are often elevated and their action is thought to modify many obesity-related diseases, including reproductive functioning.

One such adipose-derived hormone is leptin, which is best known as a regulator of food intake and energy expenditure through hypothalamus-mediated effects $[30,31]$. Although normal levels of leptin are required for overall reproductive health, excess leptin may be an important contributor to the development of reduced androgens in male obesity [32]. In addition to a higher prevalence of infertility, obese individuals are reported to have higher circulating levels of leptin than nonobese individuals [33, 34]. An increasing body of data suggests that leptin is also involved in glucose metabolism, as well as in normal sexual maturation and reproduction [35]. Leptin receptors are not only present in testicular tissue but also on the plasma membrane of sperm, suggesting that leptin may directly affect sperm through the endocrine system, independent of changes in the HPG axis $[32,36]$. Diet-induced obesity in mice caused a significant reduction in male fertility and resulted in a fivefold increase in leptin levels compared with control mice. Sperm from these obese males exhibited decreased motility and reduced hyperactivated progression compared with the lean mice [37].
As mentioned previously, adipocytes secrete various adipokines (for example, tumor necrosis factor $\alpha(\mathrm{TNF} \alpha)$, interleukin 6 (IL-6), plasminogen activator inhibitor-1 and tissue factor) [30]. A number of these adipokines have been linked to infertility and even testicular cancer. Bialas et al. [38] found that changes in the activity of intratesticular cytokines may promote various distinct pathologies such as testicular cancer or infertility. In addition, increased release of adipokines from excess white adipose tissue, resulting in inflammation, can have a toxic effect on spermatozoa through the release of excess reactive oxygen species (ROS) and reactive nitrogen species (RNS) [39]. ROS and RNS are free radicals, highly reactive and unstable molecules that arise as a consequence of oxidative stress or inflammation that can induce significant cellular damage throughout the body. It was shown that two adipocyte-released adipokines (TNF $\alpha$ and IL-6) significantly reduced human sperm progressive motility in a dose- and time-dependent manner by promoting the elevation of nitric oxide production to pathological levels [40]. Numerous authors have noted that obesity and several of its causative agents, namely, insulin resistance and dyslipidemia, are associated with increased oxidative stress [41, 42]. This association is most likely the result of elevated metabolic rates that are required to maintain normal biological processes and an increased level of stress in the local testicular environment, both of which naturally produce ROS. The local influences of biologically active substances (cytokines) released by activated leukocytes in the course of an inflammatory response to obesity may damage sperm and inhibit spermatogenesis. Agarwal et al. [43] found that abnormal patterns of increased ROS were associated with male-factor infertility and can be responsible for abnormal sperm concentration, motility and morphology found in obese males.

In a study by Winters et al. [44] the levels of inhibin B, another hormone involved in the HPG axis, declined with increasing obesity in young adult men, and values were $26 \%$ lower in men who were obese compared with normal weight men. As it was shown that inhibin B is positively correlated with the number of Sertoli cells in normal adult rhesus monkeys [45], the reduced levels of inhibin B in the Winters' study may indicate that obese men have fewer Sertoli cells than men of normal weight. As each Sertoli cell is thought to support a finite number of germ cells, fewer Sertoli cells, as a result of obesity, may result in a lower sperm count [44]. 


\subsection{Environmental toxins}

Most environmental toxins are fat-soluble and therefore accumulate in fatty tissue. Their accumulation not only around the scrotum and testes but also elsewhere in the body may disrupt the normal hormone profile, because they are proven endocrine disruptors in male fertility [19]. As morbidly obese males present with excess scrotal fat, environmental toxins accumulating in white adipose tissue surrounding the scrotum may also have a direct localized effect on spermatogenesis in the testes. Lipophilic contaminants such as organochlorides, organic compounds containing at least one covalently bonded chlorine atom whose uses are controversial because of the often toxic effects of these compounds on the environment, are associated with decreased sperm production and thus decreased male reproductive potential even if fat is not localized in the scrotal area [26]. Other toxic species that may induce abnormal spermatogenesis are ROS discussed in the previous section. Despite reports that certain toxins can negatively affect fertility, Magnusdottir et al. [7] found that poor semen quality was found to be associated with sedentary work and obesity, but not with plasma levels of persistent organochlorines.

\subsection{Physical mechanisms}

Many obese men face physical problems that could be related to their decreased fecundity and fertility, including ED, scrotal lipomatosis, which leads to increased scrotal temperatures, and sleep apnea that can cause disruptions in the nightly testosterone rise.

Despite obesity being associated with a high prevalence of ED, the pathophysiological link between obesity and ED is not clearly understood. In a recent review published in 2009, Traish et al. [14] hypothesized that visceral obesity, a component of the metabolic syndrome, increases proinflammatory factors thereby promoting the inflammatory responses and contributing to endothelial dysfunction. Any factor that affects endothelial dysfunction and impairs endothelial nitric oxide release and the integrity of the vascular bed will certainly contribute to ED, as an erection depends on hemodynamics and vascular health. Obesity is furthermore associated with reduced plasma testosterone levels, thereby contributing to hypogonadism and ED [14, 46]. In addition, there are reported relationships between low serum testosterone concentrations and ED in obese patients and those with metabolic syndrome and type II diabetes mellitus.
They found negative correlations between BMI and self-reported satisfaction with erection and BMI and the percentage of men reporting full erection [47].

Obese men, especially morbidly obese, have a higher level of scrotal fat and decreased levels of physical activity and, overall, a more sedentary lifestyle that may lead to increased testicular temperature. Studies of cyclists, truck drivers and individuals who almost constantly experience elevated scrotal temperatures show a negative influence of genital heat stress on spermatogenesis. Genital heat stress is a significant source of increased scrotal skin temperature, and even moderate and physiological elevation in scrotal skin temperature is associated with a substantially reduced sperm concentration [48]. A sedentary lifestyle should be considered as an important potential confounder for reduced sperm count.

Sleep apnea is a disorder affecting $4 \%$ of middleaged men. The disorder is characterized by repetitive collapse of the pharyngeal airway during sleep, resulting in hypoxia and hypercapnia. About twothirds of middle-aged men with obstructive sleep apnea suffer from obesity, particularly central obesity [49]. As sleep apnea disrupts the nightly rise in testosterone needed for normal spermatogenesis, it is associated with decreased pituitary-gonadal function. Therefore, sleep apnea stemming from obesity could be a further explanation for male-factor infertility and abnormal seminal parameters [50].

\section{$5 \quad$ Are there solutions?}

From the preceding literature, it is evident that obesity is an influencing factor of male infertility. Many experts believe that overweight and obesity, as well as their related chronic diseases, are largely preventable and measures can be taken to reverse both the unhealthy consequences associated with obesity and the negative impacts on male fertility. Therefore, the treatment approach will predominantly focus on the management of obesity (Figure 2). The rapid rise in the incidence of obesity has prompted researchers to not only look at natural treatment methods but also for new treatments to manage the pandemic and its subsequent comorbidities.

\subsection{Lifestyle changes}

Lifestyle changes that can lead to weight loss include diet modifications (eating smaller meals, 
cutting down on certain types of food), as well as making a conscious effort to exercise more in order to achieve a normal energy balance. Several studies showed that natural weight loss through diet and/or exercise resulted in an increase in androgen, inhibin B and sex hormone-binding globulin levels and decreased serum concentrations of insulin and leptin, thereby improving semen parameters in obese men [22, 32, 51-53]. In addition, reducing adipose tissue mass through weight loss, in association with exercise or a low-energy and lowfat diet, can decrease TNF $\alpha$, IL- 6 and other inflammatory cytokine levels associated with infertility $[54,55]$.

Gradual weight loss is best achieved through a sensible eating plan that can be maintained over long periods of time. The likelihood of maintaining weight loss is increased when the diet is combined with regular exercise, cognitive behavior therapy and a connection with a supportive group environment [56]. Therefore, adoption of these principles in a primary healthcare setting can aid in the treatment of infertility related to obesity.

\subsection{Pharmacological interventions}

Medication can be used to either treat obesity by addressing weight loss or dealing with the obesity-related effects on the male reproductive system. Currently, only two anti-obesity medications are approved by the Food and Drug Administration for long-term use [57]. One is orlistat (Xenical), which reduces intestinal fat absorption by inhibiting pancreatic lipase; the other is sibutramine (Meridia), which acts on the brain to inhibit

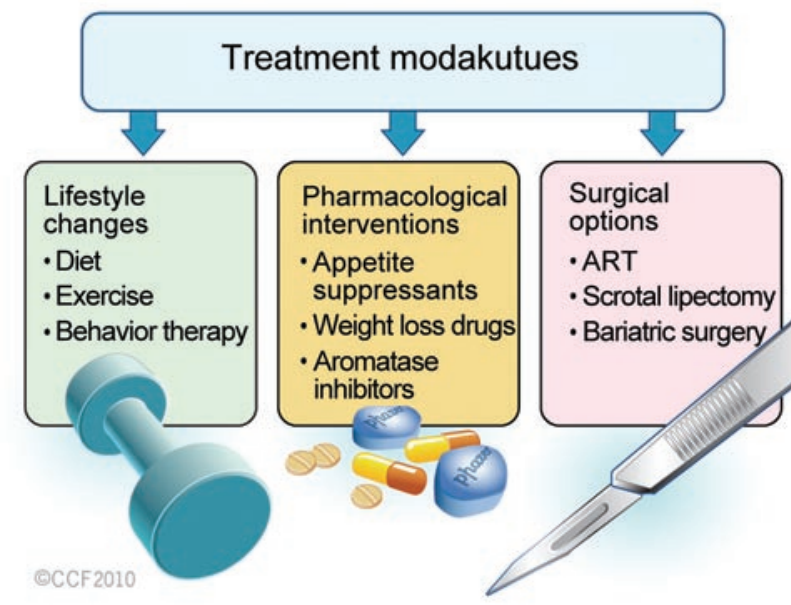

Figure 2. Approaches to treat obesity-induced male infertility. deactivation of the neurotransmitters norepinephrine, serotonin and dopamine, therefore decreasing appetite. Weight loss with these drugs is however modest.

Aromatase inhibitors are an option for obese males facing infertility problems, especially if they have elevated estrogen and lowered testosterone levels. Aromatase inhibitors prevent the excess aromatase enzymes from converting testosterone to estrogen. Numerous case studies have found this to be an effective treatment in not only restoring normal hormone levels but also fertility. Zumoff et al. [58] found that by inhibiting estrogen biosynthesis (through administration of the aromatase inhibitor testolactone), there was an alleviation of possible infertility as a result of hypogonadotropic hypogonadism of the obese male subjects. In another case study, a patient diagnosed with infertility secondary to morbid obesity was treated with the aromatase inhibitor, anastrozole. This led to normalization of the patient's testosterone, LH and FSH hormone levels, as well as suppression of the serum estradiol levels and the normalization of spermatogenesis and fertility [27]. In a study including normal, overweight and obese men, treatment with anastrozole led to an increase in testosteroneto-estradiol ratio that occurred in association with increased semen parameters. Anastrozole and testolactone have similar effects on hormonal profiles and semen analysis, but anastrazole seems to be at least as effective as testolactone for treating men with abnormal testosterone-to-estradiol ratio [59].

New directions in pharmacological treatment might include testosterone replacement therapy and maintenance and regulation of adipose-derived hormones, particularly leptin, which is produced by fat cells in the body and is known to affect appetite and the body's energy balance, while also related to reproductive function. Testosterone replacement therapy has been shown to suppress the levels of circulating leptin, although no information regarding the effect of the treatment on semen parameters was reported [32, 35]. Quennell et al. [60] discovered that leptin indirectly regulates gonadotropin-releasing hormone's neuronal function, affecting forebrain neurons that induce infertility. By decreasing elevated leptin levels in obese patients, it might be possible to reverse some of the potential suppressive effects of excess leptin on the HPG axis and restore normal spermatogenesis and sperm function. Ghrelin, a hormone that is secreted by cells in the lining of the 
stomach, also affects appetite and the body's energy balance. Further studies of these hormones may lead to the development of new medications to control appetite and provide an option for treating obesity-related health problems and infertility.

\subsection{Surgical options}

In vitro fertilization (IVF) may be an option for obese patients facing problems such as ED or other purely physical fertility problems. Although morbid obesity is associated with unfavorable IVF/ intracytoplasmic sperm injection cycle outcome, as evidenced by lower pregnancy rates in females, there is no evidence for a contributing male factor when assisted reproductive methods are used [61]. It is recommended that morbidly obese patients undergo appropriate counseling before the initiation of this expensive and invasive therapy. Fortunately, studies show that obesity in men may not adversely affect the results of their partners who are undergoing IVF or embryo transfer [62].

Scrotal lipectomy is a treatment option available for infertility in obese men whose excess fat accumulation may be contributing to their infertility, either through increased scrotal temperature or excess toxin accumulation. Onefifth of patients who were previously considered infertile and underwent scrotal lipectomy to remove excess fat were able to achieve a successful pregnancy [22].

For individuals who are severely obese, dietary changes and behavior modification may be accompanied by surgery to reduce or bypass portions of the stomach or small intestine. The risks of obesity surgery have declined in recent years, but it is still only performed on patients for whom other strategies have failed and whose obesity seriously threatens their health.

Bariatric surgery ('weight loss surgery') is the use of surgical intervention in the treatment of obesity by reducing or bypassing portions of the stomach or small intestine. As it is a rather extreme intervention, it is only recommended for severely obese people (BMI $>40$ ) who have failed to lose sufficient weight after dietary modification and pharmacological treatment [63]. Gastric bypass and banding surgeries are very effective in the treatment of morbid obesity and its comorbid conditions. One study reported that a significant decrease in estrogen, increase in testosterone and normalization of other hormone and adipokine levels were experienced by patients who underwent vertical banded gastroplasty [64]. However, others speculate that the drastic weight loss that accompanies this procedure might induce secondary infertility, even though natural weight loss has shown promising results in terms of restoring fertility [65]. Bariatric surgery should therefore not be recommended as a treatment for obesity-linked infertility until extensive, long-term studies have been performed to determine the definite effects on male fertility.

\section{Conclusion}

Obesity is a modern-day pandemic with serious comorbidities, both physical and psychological. Studies clearly show that obese men have an increased chance of subfertility and subfecundity due to various mechanisms (physical, genetic, hormonal, adipokine, cytokine) that ultimately lead to ED and abnormal semen parameters. The central factor to all of these mechanisms is the abnormal regulation of the HPG axis. An abnormal hormonal profile and, more specifically, increased adipose-derived hormones and adipokine levels may explain the association between BMI, altered semen parameters and infertility more accurately, as it is clearly more complex than that can be accounted for simply by abnormal levels of reproductive hormones.

New studies point to many causes for abnormal semen parameters, including genetic markers, excess adipose-derived hormone and adipokine release, as well as oxidative stress. The consistent decrease in inhibin B levels and increase in leptin levels, and specific proteomic sperm changes observed in obese infertile males, all may have negative impacts on spermatogenesis. Increased cytokines, such as IL-6, are connected with oxidative stress and impaired reproduction, and could also contribute to abnormal semen parameters observed in obese men facing fertility issues. These markers point toward a true suppression of normal spermatogenesis and sperm quality, despite some inconsistency in the results of studies performed to measure the effects of obesity on semen parameters.

In treating obesity-linked sperm disorders and male infertility, few controlled studies have been performed, and effective therapeutic treatments, advice for lifestyle changes and surgical options should be explored further. In addition, determining the most accurate measure for qualifying patients as obese could more accurately clarify the cause of their infertility and other health issues that might accompany their state of obesity. The increasing 
prevalence of obesity all over the world in conjunction with a perceived declining male sperm count in modern man calls for more research and attention to obesity as an etiology of male infertility. Clinicians should therefore definitely consider obesity when a male patient with idiopathic infertility is confronted.

\section{References}

1 World Health Organization. WHO Technical Report: Diet, Nutrition and the Prevention of Chronic Disease. WHO 2003; Retrieved from www.who/int/en.

2 Popkin BM. Recent dynamics suggest selected countries catching up to US obesity. Am J Clin Nutr 2010; 91: 284-8.

3 World Health Organization. Obesity and overweight; 2006. Retrieved from www.who/int/en.

4 Swan SH, Elkin EP, Fenster L. The question of declining sperm density revisited: an analysis of 101 studies published 1934-1996. Environ Health Perspect 2000; 108: 961-6.

5 Sallmen M, Sandler DP, Hoppin JA, Blair A, Baird DD. Reduced fertility among overweight and obese men. Epidemiology 2006; 17: 520-3.

6 Carlsen E, Giwercman A, Keiding N, Skakkebaek NE. Evidence for decreasing quality of semen during past 50 years. BMJ 1992; 305: 609-13.

7 Magnusdottir EV, Thorsteinsson T, Thorsteinsdottir S, Heimisdottir M, Olafsdottir K. Persistent organochlorines, sedentary occupation, obesity and human male subfertility. Hum Reprod 2005; 20: 208-15.

8 Kyle UG, Bosaeus I, De Lorenzo AD, Deurenberg P, Elia $\mathrm{M}$, et al. Composition of the ESPEN Working Group. Bioelectrical impedance analysis-part I: review of principles and methods. Clin Nutr 2004; 23: 1226-43.

9 Booth ML, Hunter C, Gore CJ, Bauman A, Owen N. The relationship between body mass index and waist circumference: implications for estimates of the population prevalence of overweight. Int J Obes Relat Metab Disord 2000; 24: 1058-61.

10 Huxley R, Mendis S, Zheleznyakov E, Reddy S, Chan J. Body mass index, waist circumference and waist:Hip ratio as predictors of cardiovascular risk-a review of the literature. Eur J Clin Nutr 2010; 64: 16-22.

11 Akpinar E, Bashan I, Bozdemir N, Saatci E. Which is the best anthropometric technique to identify obesity: Body mass index, waist circumference or waist-hip ratio? Coll Antropol 2007; 31: 387-93.

12 Adams JP, Murphy PG. Obesity in anaesthesia and intensive care. Br J Anaesth 2000; 85: 91-108.

13 Kushner RF, Bessesen DH. Contemporary Endocrinology: Treatment of the Obese Patient. Humana Press Inc., Totowa, NJ, p1-26.

14 Traish AM, Feeley RJ, Guay A. Mechanisms of obesity and related pathologies: androgen deficiency and endothelial dysfunction may be the link between obesity and erectile dysfunction. FEBS J 2009; 276: 5755-67.

15 Corona G, Mannucci E, Schulman C, Petrone L, Mansani R, et al. Psychobiologic correlates of the metabolic syndrome and associated sexual dysfunction. Eur Urol 2006; 50:
595-604; discussion 604.

16 Ramlau-Hansen CH, Thulstrup AM, Nohr EA, Bonde JP, Sorensen TI, et al. Subfecundity in overweight and obese couples. Hum Reprod 2007; 22: 1634-7.

17 Jensen TK, Andersson AM, Jorgensen N, Andersen AG, Carlsen E, et al. Body mass index in relation to semen quality and reproductive hormones among 1,558 danish men. Fertil Steril 2004; 82: 863-70.

18 Martini AC, Tissera A, Estofan D, Molina RI, Mangeaud A, et al. Overweight and seminal quality: a study of 794 patients. Fertil Steril 2010; Jan 5. [Epub ahead of print].

19 Hammoud AO, Wilde N, Gibson M, Parks A, Carrell DT, et al. Male obesity and alteration in sperm parameters. Fertil Steril 2008; 90: 2222-5.

20 Smit M, Romijn JC, Wildhagen MF, Weber RF, Dohle GR. Sperm chromatin structure is associated with the quality of spermatogenesis in infertile patients. Fertil Steril 2009. [Epub ahead of print].

21 Kriegel TM, Heidenreich F, Kettner K, Pursche T, Hoflack B, et al. Identification of diabetes- and obesity-associated proteomic changes in human spermatozoa by difference gel electrophoresis. Reprod Biomed Online 2009; 19: 660-70.

22 Kasturi SS, Tannir J, Brannigan RE. The metabolic syndrome and male infertility. J Androl 2008; 29: 251-9.

23 Arsov T, Silva DG, O’Bryan MK, Sainsbury A, Lee NJ, et al. Fat aussie - a new alstrom syndrome mouse showing a critical role for ALMS1 in obesity, diabetes, and spermatogenesis. Mol Endocrinol 2006; 20: 1610-22.

24 Hammoud A, Carrell DT, Meikle AW, Xin Y, Hunt SC, et al. An aromatase polymorphism modulates the relationship between weight and estradiol levels in obese men. Fertil Steril 2009. [Epub ahead of print].

25 Strain GW, Zumoff B, Kream J, Strain JJ, Deucher R, et al. Mild hypogonadotropic hypogonadism in obese men. Metabolism 1982; 31: 871-5.

26 Tsai EC, Matsumoto AM, Fujimoto WY, Boyko EJ. Association of bioavailable, free, and total testosterone with insulin resistance: influence of sex hormone-binding globulin and body fat. Diabetes Care 2004; 27: 861-8.

27 Roth MY, Amory JK, Page ST. Treatment of male infertility secondary to morbid obesity. Nat Clin Pract Endocrinol Metab 2008; 4: 415-9.

28 Qin DD, Yuan W, Zhou WJ, Cui YQ, Wu JQ, et al. Do reproductive hormones explain the association between body mass index and semen quality? Asian J Androl 2007; 9: 827-34.

29 Athyros VG, Tziomalos K, Karagiannis A, Anagnostis P, Mikhailidis DP. Should adipokines be considered in the choice of the treatment of obesity-related health problems? Curr Drug Targets 2010; 11: 122-35.

30 Trayhurn P, Beattie JH. Physiological role of adipose tissue: white adipose tissue as an endocrine and secretory organ. Proc Nutr Soc 2001; 60: 329-39.

31 Bhat GK, Sea TL, Olatinwo MO, Simorangkir D, Ford $\mathrm{GD}$, et al. Influence of a leptin deficiency on testicular morphology, germ cell apoptosis, and expression levels of apoptosis-related genes in the mouse. J Androl 2006; 27 : 302-10.

32 Isidori AM, Caprio M, Strollo F, Moretti C, Frajese G, et al. Leptin and androgens in male obesity: evidence for leptin contribution to reduced androgen levels. J Clin Endocrinol 
Metab 1999; 84: 3673-80.

33 Wang P, Mariman E, Renes J, Keijer J. The secretory function of adipocytes in the physiology of white adipose tissue. J Cell Physiol 2008; 216: 3-13.

34 Wozniak SE, Gee LL, Wachtel MS, Frezza EE. Adipose tissue: the new endocrine organ? A review article. Dig Dis Sci 2009; 54: 1847-56.

35 Hofny ER, Ali ME, Abdel-Hafez HZ, El-Dien Kamal E, Mohamed EE, et al. Semen parameters and hormonal profile in obese fertile and infertile males. Fertil Steril 2009. [Epub ahead of print].

36 Jope T, Lammert A, Kratzsch J, Paasch U, Glander HJ. Leptin and leptin receptor in human seminal plasma and in human spermatozoa. Int J Androl 2003; 26: 335-41.

37 Ghanayem BI, Bai R, Kissling GE, Travlos G, Hoffler U. Dietinduced obesity in male mice is associated with reduced fertility and potentiation of acrylamide-induced reproductive toxicity. Biol Reprod 2010; 82: 96-104.

38 Bialas M, Fiszer D, Rozwadowska N, Kosicki W, Jedrzejczak P, et al. The role of IL-6, IL-10, TNF-alpha and its receptors TNFR 1 and TNFR2 in the local regulatory system of normal and impaired human spermatogenesis. Am J Reprod Immunol 2009; 62: 51-9.

39 Fraczek M, Kurpisz M. Inflammatory mediators exert toxic effects of oxidative stress on human spermatozoa. J Androl 2007; 28: 325-33.

40 Lampiao F, du Plessis SS. TNF-alpha and IL-6 affect human sperm function by elevating nitric oxide production. Reprod Biomed Online 2008; 17: 628-31.

41 Dandona P, Aljada A, Chaudhuri A, Mohanty P, Garg R. Metabolic syndrome: a comprehensive perspective based on interactions between obesity, diabetes, and inflammation. Circulation 2005; 111: 1448-54.

42 Davi G, Falco A. Oxidant stress, inflammation and atherogenesis. Lupus 2005; 14: 760-4.

43 Agarwal A, Sharma RK, Nallella KP, Thomas AJ Jr, Alvarez $\mathrm{JG}$, et al. Reactive oxygen species as an independent marker of male factor infertility. Fertil Steril 2006; 86: 878-85.

44 Winters SJ, Wang C, Abdelrahaman E, Hadeed V, Dyky $\mathrm{MA}$, et al. Inhibin-B levels in healthy young adult men and prepubertal boys: is obesity the cause for the contemporary decline in sperm count because of fewer sertoli cells? J Androl 2006; 27: 560-4.

45 Ramaswamy S, Marshall GR, McNeilly AS, Plant TM. Evidence that in a physiological setting sertoli cell number is the major determinant of circulating concentrations of inhibin $\mathrm{B}$ in the adult male rhesus monkey (macaca mulatta). J Androl 1999; 20: 430-4.

46 Feeley RJ, Traish AM. Obesity and erectile dysfunction: is androgen deficiency the common link? Scientific WorldJournal 2009; 9: 676-84.

47 Diaz-Arjonilla M, Schwarcz M, Swerdloff RS, Wang C. Obesity, low testosterone levels and erectile dysfunction. Int J Impot Res 2009; 21: 89-98.

48 Hjollund NH, Bonde JP, Jensen TK, Olsen J. Diurnal scrotal skin temperature and semen quality. The danish first pregnancy planner study team. Int J Androl 2000; 23: 309-18.

49 Vgontzas AN, Papanicolaou DA, Bixler EO, Hopper K, Lotsikas A, et al. Sleep apnea and daytime sleepiness and fatigue: relation to visceral obesity, insulin resistance, and hypercytokinemia. J Clin Endocrinol Metab 2000; 85:
$1151-8$.

50 Luboshitzky R, Lavie L, Shen-Orr Z, Herer P. Altered luteinizing hormone and testosterone secretion in middleaged obese men with obstructive sleep apnea. Obes Res 2005; 13: 780-6.

51 Chavarro JE, Toth TL, Wright DL, Meeker JD, Hauser R. Body mass index in relation to semen quality, sperm DNA integrity, and serum reproductive hormone levels among men attending an infertility clinic. Fertil Steril 2010; 93: 2222-31.

52 Kaukua J, Pekkarinen T, Sane T, Mustajoki P. Sex hormones and sexual function in obese men losing weight. Obes Res 2003; 11: 689-94.

53 Niskanen L, Laaksonen DE, Punnonen K, Mustajoki P, Kaukua J, et al. Changes in sex hormone-binding globulin and testosterone during weight loss and weight maintenance in abdominally obese men with the metabolic syndrome. Diabetes Obes Metab 2004; 6: 208-15.

54 Sharman MJ, Volek JS. Weight loss leads to reductions in inflammatory biomarkers after a very-low-carbohydrate diet and a low-fat diet in overweight men. Clin Sci (Lond) 2004; 107: 365-9.

55 Ziccardi P, Nappo F, Giugliano G, Esposito K, Marfella R, et al. Reduction of inflammatory cytokine concentrations and improvement of endothelial functions in obese women after weight loss over one year. Circulation 2002; 105: 804-9.

56 Lau DC, Douketis JD, Morrison KM, Hramiak IM, Sharma AM, et al. Obesity Canada Clinical Practice Guidelines Expert Panel. 2006 Canadian clinical practice guidelines on the management and prevention of obesity in adults and children [summary]. CMAJ 2007; 176: 1-13.

57 Prescription medications for the treatment of obesity [Internet]. Available from: http://win.niddk.nih.gov/publications/ prescription.htm\#fdameds.

58 Zumoff B, Miller LK, Strain GW. Reversal of the hypogonadotropic hypogonadism of obese men by administration of the aromatase inhibitor testolactone. Metabolism 2003; 52: 1126-8.

59 Raman JD, Schlegel PN. Aromatase inhibitors for male infertility. J Urol 2002; 167: 624-9.

60 Quennell JH, Mulligan AC, Tups A, Liu X, Phipps SJ, et al. Leptin indirectly regulates gonadotropin-releasing hormone neuronal function. Endocrinology 2009; 150: 2805-12.

61 Awartani KA, Nahas S, Al Hassan SH, Al Deery MA, Coskun S. Infertility treatment outcome in sub groups of obese population. Reprod Biol Endocrinol 2009; 7: 52.

62 Dechaud H, Anahory T, Reyftmann L, Loup V, Hamamah $\mathrm{S}$, et al. Obesity does not adversely affect results in patients who are undergoing in vitro fertilization and embryo transfer. Eur J Obstet Gynecol Reprod Biol 2006; 127: 88-93.

63 Encinosa WE, Bernard DM, Chen CC, Steiner CA. Healthcare utilization and outcomes after bariatric surgery. Med Care 2006; 44: 706-12.

64 Bastounis EA, Karayiannakis AJ, Syrigos K, Zbar A, Makri GG, et al. Sex hormone changes in morbidly obese patients after vertical banded gastroplasty. Eur Surg Res 1998; 30: 43-7.

65 di Frega AS, Dale B, Di Matteo L, Wilding M. Secondary male factor infertility after roux-en-Y gastric bypass for morbid obesity: case report. Hum Reprod 2005; 20: 997-8. 